Finnish Centre for Radiation and Nuclear Safety and Finnish Cancer Registry, Helsinki, Finland

Anssi Auvinen, senior scientist Timo Hakulinen, professor Kristina Servomaa, laboratory head

Tapio Rytömaa, research director

\section{Extramural Programs} Branch and Radiation Epldemiology Branch, National Cancer Institute, Bethesda, MD, USA G Iris Obrams, chief

John D Boice Jr, chief

Correspondence to:

Dr Bigbee. matched for age. The data, summarised in table 1 , show a tendency for increased frequency of variants with loss of the glycophorin A allele in all three groups of workers compared with the controls, although none of the differences is significant. The pooled results, with an average increase of frequency of $\sim 1-2 \times 10^{-6}$ corresponding to radiation doses of $\sim 4-8 \mathrm{cGy},{ }^{4}$ indicate that these workers' average exposure was unlikely to greatly exceed 10-20 cGy, the approximate minimum radiation dose detectable by our assay.

\section{Comment}

We undertook this biodosimetry study to ascertain whether many Chernobyl cleanup workers received substantial radiation exposures that were either undocumented or inaccurately recorded. Our initial biodosimetry data strongly suggest that this is unlikely. It also seems that there is not a large subset of these workers who received doses substantially above the average physical doses. Thus the estimates of physical doses, while perhaps incomplete and imprecise, cannot be rejected as inadequately characterising the workers' exposures. Our results support the use of these estimates to assess possible health hazards and as the basis of power calculations for epidemiological studies of populations of Chernobyl cleanup workers. To strengthen this conclusion, we are now performing fluo- rescence in situ hybridisation (FISH) based chromosomal translocation analysis in the peripheral blood lymphocytes of these workers. We are also studying the incidence of leukaemia and prevalence of thyroid cancer and are constructing assessments of exposure using combined physical dosimetry records, extensive questionnaire data, and biological dosimetry methods.

Funding: This work was supported by Contract No N01-CP-50520 from the Radiation Epidemiology Branch of the US National Cancer Institute.

Conflict of interest: None.

1 United Nations Scientific Committee on the Effects of Atomic Radiation (UNSCEAR). Sources, effects and risk of ionizing radiation. 1988 Report to the general assembly, with annexes (E.88.IX.7). New York, NY: United Nations, 1988.

2 Jensen RH, Bigbee WL. Direct immunofluorescence labeling provides an improved method for the glycophorin A somatic cell mutation assay. Cyrometry (in press).

3 Langlois RG, Akiyama M, KusunokjY, Bigbee WL, Grant SG, DuPont BR, et al. Analysis of somatic mutations at the glycophorin $A$ locus in atomi bomb survivors: a comparative study of assay methods. Radiat Res 1993;136:111-7.

4 Jensen RH, Langlois RG, Bigbee WL, Grant SG, Moore D II, Pilinskaya M, et al. Elevated frequency of glycophorin A mutations in erythrocytes from Chemobyl accident victims. Radiat Res 1995;141:129-35.

5 Straume T, Langlois RG, Lucas J, Jensen RH, Bigbee WL, Ramalho AT et al. Novel biodosimetry methods applied to victims of the Goiânia accident. Health Phys 1991;60:71-6.

(Accepted 9 April 1996)

\title{
Transformations, means, and confidence intervals
}

\section{J Martin Bland, Douglas G Altman}

This is the 18th in a series of occasional notes on medical statistics

\section{Department of Public}

Health Sciences,

St George's Hospital

Medical School, London SW17 0RE

J Martin Bland, professor of

medical statistics

ICRF Medical Statistics Group, Centre for

Statistics in Medicine, Institute of Health

Sclences, PO Box 777,

Oxford OX3 7LF

Douglas G Altman, head

Correspondence to:

Professor Bland.

BMf 1996;312:1079
When we use transformed data in analyses, ${ }^{1}$ this affects the final estimates that we obtain. Figure 1 shows some serum triglyceride measurements, which have a skewed distribution. A logarithmic transformation is often useful for data which have positive skewness like this, and here the approximation to a normal distribution is greatly improved. For the untransformed data the mean is $0.51 \mathrm{mmol} / \mathrm{l}$ and the standard deviation $0.22 \mathrm{mmol} / \mathrm{l}$. The mean of the $\log _{10}$ transformed data is -0.33 and the standard deviation is 0.17 . If we take the mean on the transformed scale and back transform by taking the antilog, we get $10^{-0.33}=0.47 \mathrm{mmol} / 1$. We call the value estimated in this way the geometric mean. The geometric mean will be less than the mean of the raw data.

When triglyceride is measured in $\mathrm{mmol} / \mathrm{l}$ the $\mathrm{log}$ of a single observation is the $\log$ of a measurement in $\mathrm{mmol} / \mathrm{l}$. The average of $n$ such transformed measurements is also the $\log$ of a number in $\mathrm{mmol} / \mathrm{l}$, so the anti$\log$ is back in the original units, $\mathrm{mmol} / \mathrm{l}$.

The antilog of the standard deviation, however, is not measured in $\mathrm{mmol} /$. Calculation of the standard deviation of the $\log$ transformed data requires taking the difference between each log observation and the log geometric mean. The difference between the log of two numbers is the $\log$ of their ratio. ${ }^{2}$ As a ratio is a dimensionless pure number, the units in which serum triglyceride was measured would not matter; the standard deviation on the log scale would be the same. As a result, we cannot transform the standard deviation back to the original scale.

If we want to use the standard deviation or standard error it is easiest to do all calculations on the transformed scale and transform back, if necessary, at the end. For example, the $95 \%$ confidence interval for the mean on the $\log$ scale is -0.35 to -0.31 . To get back to the original scale we antilog the confidence limits on the log scale to give a

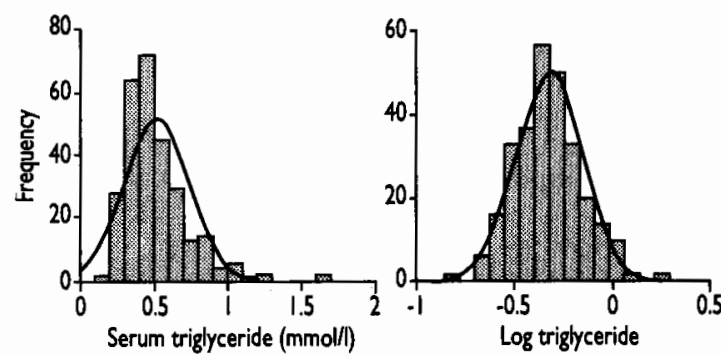

Fig 1 -Serum triglyceride and $\log _{10}$ serum triglyceride concentrations in cord blood for 282 babies, with best fitting normal distribution

$95 \%$ confidence interval for the geometric mean on the natural scale $(0.47)$ of 0.45 to $0.49 \mathrm{mmol} / 1$. For comparison, the $95 \%$ confidence interval for the arithmetic mean using the raw, untransformed data is 0.48 to $0.54 \mathrm{mmol} / \mathrm{l}$ These limits are wider than those for the geometric mean. This is because with highly skewed data the extreme observations have a large influence on the arithmetic mean, making it more prone to sampling error. Lessening this influence is one advantage of using transformed data.

If we use another transformation, such as the reciprocal or the square root, ${ }^{1}$ the same principle applies. We carry out all calculations on the transformed scale and transform back once we have calculated the confidence interval. This works for the sample mean and its confidence interval. Things become more complicated if we look at the difference between two means. We shall look at this in another Statistics Note.

1 Bland JM, Altman DG. Transforming data. BMF 1996;312:770. 2 Bland JM, Altman DG. Logarichms. BMY I996;312:700. 